\title{
Recanalization and Mortality Rates of Thrombectomy With Stent-Retrievers in Octogenarian Patients with Acute Ischemic Stroke
}

\author{
G. Parrilla $\cdot$ E. Carreón - J. Zamarro • M. Espinosa de Rueda \\ B. García-Villalba · F. Marín • F. Hernández-Fernández • \\ A. Morales • M. Fernández-Vivas • R. Núñez $\cdot$ A. Moreno
}

Received: 2 January 2014/ Accepted: 21 April 2014/Published online: 13 June 2014

(C) Springer Science+Business Media New York and the Cardiovascular and Interventional Radiological Society of Europe (CIRSE) 2014

\begin{abstract}
Background Our objective was to evaluate the effect of treatment with stent-retrievers in octogenarians suffering an acute ischemic stroke.

Methods A total of 150 consecutive patients with acute stroke who were treated with stent-retrievers between April 2010 and June 2012 were retrospectively reviewed. Patients were divided into those $<80$ years old $(n=116)$ and those $\geq 80(n=34)$. Baseline characteristics, procedure data, and endpoints (postprocedural NIHSS, death, and mRS at 3 months) were compared.

Results High blood pressure, atrial fibrillation, and anticoagulation were more frequent in octogenarians $(p=0.01$, 0.003 , and 0.04 respectively). There were no differences between both groups regarding previous intravenous thrombolysis (32.4 vs. $48.3 \%, p=0.1$ ), preprocedural NIHSS (18.1 vs. $16.8, p=0.3)$, procedure time $(74.5$ (40-114) $\min$ vs. 63 (38-92) $\mathrm{min}, p=0.2)$, revascularization time (380.5 (298-526.3) min vs. 350 (296.3-452.8),
\end{abstract}

G. Parrilla $(\bowtie) \cdot$ J. Zamarro · M. Espinosa de Rueda .

B. García-Villalba · F. Hernández-Fernández · A. Moreno

Service of Interventional Neuroradiology, Hospital Clínico

Universitario Virgen de la Arrixaca, Carretera Madrid-Cartagena

S/N, 30120 Murcia, Spain

e-mail: gpr1972@gmail.com

E. Carreón · A. Morales

Service of Neurology Hospital Clínico Universitario Virgen de la Arrixaca, Murcia, Spain

F. Marín

Department of Cardiology, Hospital Clínico Universitario

Virgen de la Arrixaca, Murcia, Spain

M. Fernández-Vivas · R. Núñez

Intensive Care Unit, Hospital Clínico Universitario Virgen de la

Arrixaca, Murcia, Spain $p=0.3)$, TICI $\geq 2 \mathrm{~B}$ (88.2 vs. $93.9 \%, p=0.1)$, and symptomatic haemorrhage ( 5.9 vs. $2.6 \%, p=0.3$ ). Discharge NIHSS was higher in octogenarians (9.7 vs. $6.5, p=$ $0.03)$. Death and 3-month $\mathrm{mRS} \geq 3$ were more frequent in octogenarians (35.3 vs. $17.2 \%, p=0.02$ and 73.5 vs. $37.1 \%, p=0.02)$. ICA-involvement and prolonged revascularization involved higher mortality (66.7 vs. $27.6 \%, p=0.03$ ) and worse mRS (50 vs. $24.4 \%, p=0.06$ ) in octogenarians.

Conclusions In our series, treatment with stent-retrievers in octogenarians with acute ischemic stroke achieved good rates of recanalization but with a high mortality rate. ICA involvement and revascularization times beyond 6 hours associated to a worse prognosis. These data might be of value in the design of prospective studies evaluating the clinical efficacy of the endovascular treatments in octogenarians.

Keywords Neurointerventions · Endovascular treatment · Stroke therapy · Thrombectomy · Brain/neurological/nervous system $\cdot$ Stroke

\section{Introduction}

During the past years, there has been a significant increase in the proportion of acute ischemic stroke patients receiving revascularization by endovascular treatment, with patients aged $\geq 80$ years having the lowest rate of utilization [1]. This tendency might be partly explained by the results of previous studies, which show that the mortality rate after different treatments (intravenous thrombolysis, intra-arterial thrombolysis, mechanical thrombectomy with classic retrievers) is higher in octogenarians than in younger patients, conveying increased age a higher rate of 
stroke-related death [2-4]. In the past years, stent-retrievers seem to have opened a new window in the field of mechanical thrombectomy, achieving better angiographic and clinical outcomes than classic retrievers [5, 6], but their effect and security in the older patients have scarcely been reported [7]. We present the results of a series of octogenarians patients who were treated with stent-retrievers, compare them with a control group, and discuss the topic of endovascular treatment of acute stroke in octogenarians.

\section{Patients and Methods}

\section{General Design of the Study}

All patients with acute stroke that were treated with mechanical thrombectomy using stent retrievers between April 2010 and June 2012 were retrospectively reviewed from our centre's stroke database. For the purpose of this study, patients were divided into those $<80$ and those $\geq 80$ years old. Baseline characteristics (age, high blood pressure, diabetes mellitus, dyslipemia, atrial fibrillation, smoking habit, anticoagulant treatment), stroke parameters (stroke subtype, occluded vessel, previous intravenous thrombolysis, preprocedural National Institute of Health Stroke Scale (NIHSS), procedure and revascularization times, and outcome (NIHSS at discharge, modified Rankin Scale at 3 months, and death) were subsequently compared.

Stroke subtype was established according to the aetiology into atherothrombotic, cardioembolic, arterial dissection, or undetermined (when no aetiology for the stroke could be found or when two or more possible etiologist were found). Location of the occluded vessel was established by catheter angiography and divided into extracranial internal carotid (ECA), intracranial internal carotid (ICA), middle cerebral artery (MCA), vertebrobasilar (VB), or tandem occlusion (TO). Procedural time was defined as the time between the groin puncture and angiographic revascularization of the vessel correspondent to $\mathrm{TICI} \geq 2 \mathrm{~B}$. Revascularization time was the time between symptom onset and an angiographic revascularization $\mathrm{TICI} \geq 2 \mathrm{~B}$.

\section{End Points}

The end point of the study was to determine whether there were differences in the clinical outcome between both groups of patients. Clinical outcome was defined by postprocedural NIHSS, death, and modified Rankin scale 3 months postprocedure. Patients were followed up by neurologists in external consultation 3 months after discharge. In those cases which in-person follow-up was not possible (patient refusal, great disability), consultation was made by phone.

\section{Patient Transferral}

Our hospital is the only endovascular stroke centre in the region, covering an area of 11,313 square kilometres and a population of approximately $1,470,000$ people. It is situated in south of the region, and the patients to be considered for mechanical treatment who do not come directly to our emergency room are transferred to our centre either from the mobile emergency units that operate through the region or from the emergency rooms of the other six hospitals in the area. In one of these centres a neurologist on call evaluates the stroke patient, and in the other five centres and mobile emergency units the initial neurologic evaluation is performed by the emergency physicians. Each case considered subsidiary of mechanical thrombectomy is communicated by phone to the staff neurologist and neurointerventionist on call at our centre, and a decision to transfer the patient or not is made. The same vehicles then transfer those selected patients that had been initially attended by mobile units to our centre, and the nearest ambulance transfers those who have been attended in the emergency units of other hospitals.

\section{Stroke Protocol and Selection Criteria}

Once in our centre, patients are selected for mechanical thrombectomy after an imaging and clinical protocol that is performed in all stroke patients who are considered candidates for intravenous thrombolysis and/or mechanical thrombectomy and includes urgent nonenhanced CT, CT angiography, and CT perfusion. Candidates for intravenous thrombolysis are selected according to the National Institute of Neurological Disorders [8] and Stroke and the Third European Cooperative Acute Stroke Study criteria [9, 10]. Indications for mechanical thrombectomy include 1) rescue therapy, performed in those patients in whom intravenous thrombolysis is not accompanied by a clinical response (improvement in NIHSS score $\geq 4$ ) in the first 60 minutes after treatment, and 2) direct thrombectomy, which is performed in those patients in whom intravenous thrombolysis is contraindicated and in those with large vessel stroke that arrive at our hospital between 4.5 and 8 hours after symptom onset [11-15].

Exclusion criteria for mechanical thrombectomy included: presence of cerebral haemorrhage, an ASPECTS score of less than 7 in anterior circulation strokes or evidence of established large cerebellar and brain stem infarction in posterior circulation strokes in the nonenhanced CT, absence of large vessel vascular occlusion in the CT angiography, or any medical conditions that preclude a general anaesthesia. For those strokes beyond 6 hours since symptom onset, a CT perfusion mismatch greater than $50 \%$ was also requested. 


\section{Procedure}

Before angiography and mechanical thrombectomy, all patients or relatives gave informed consent. All of the procedures were performed under general anaesthesia. Angiography was performed via a femoral approach using a $9 \mathrm{~F}$ sheath and a diagnostic catheter, generally a JB 4F (Cordis, Miami Lakes, FL). Once the location of the clot was identified, the diagnostic catheter was removed and an 8F balloon guide catheter (Concentric Medical, Mountain View, CA) was advanced and placed in the internal carotid artery (anterior circulation strokes) or in the vertebral artery (posterior circulation strokes). For those cases in which the placement of an $8 \mathrm{~F}$ catheter was not possible due to the diameter of the vertebral artery, a Neuron 6F (Penumbra Inc., Alameda, CA) was placed instead. A Rebar-18 microcatheter (ev3 Neurovascular, Irvine, CA) with a 0.014-inch Traxcess guidewire (MicroVention, Tustin, CA) was then advanced distally to the clot. The microwire was then removed and a stent-retriever Solitaire AB 4 or $6 \mathrm{~mm}$, FR 4 or $6 \mathrm{~mm}$ (ev3 Neurovascular), Trevo $4 \mathrm{~mm}$, Trevo Pro $4 \mathrm{~mm}$ (Concentric Medical), or Capture 3 or $4 \mathrm{~mm}$ (MindFrame, Irvine, CA) was used to engage and snare the clot. Once the clot was captured, the balloon guide catheter was inflated to temporally arrest forward flow while the clot was being withdrawn. The clot was first pulled into the catheter guide and then completely out of the body while aspirating with a 50-mL syringe. The balloon was then deflated, and the flow was restored. Recanalization was assessed on the control angiogram following TICI classification [16].

\section{Postprocedure Management}

Once the procedure was finished, the patients were admitted to the intensive care unit for 24 hours and then to the stroke unit. Antiplatelet or anticoagulation therapy was initiated after the initial postprocedural scan had ruled out symptomatic haemorrhage. The choice of therapy depended on the suspected aetiology of the stroke. Initial anticoagulation with intravenous heparin and a posterior switch to acenocoumarol was used for inferred cardioembolism in the setting of atrial fibrillation, whereas antiplatelet therapy (aspirin, $200 \mathrm{mg} /$ day, or clopidogrel, $75 \mathrm{mg} /$ day) was preferred in the setting of atherothrombotic aetiologies. Control of blood pressure, fever, hyperglycaemia, aspiration risk, deep venous thrombosis prophylaxis, and all other essential aspects in acute stroke management were performed following current stroke guidelines [17].

\section{Statistical Analyses}

Continuous variables were tested for normality by the Kolmogorov-Smirnov test. Continuous variables are presented as a mean \pm standard deviation (SD) or median (25th and 75 th percentiles), as appropriate, and categorical variables as a percentage. Univariate analyses were performed, including the Student (or Mann-Whitney $U$ test if appropriate) for the continuous variables and the Chi-square test for the categorical variables. We explored the influence of different variables on prognosis, in particular hypertension, internal carotid involvement, revascularization time beyond 6 hours, oral anticoagulation, and presence of atrial fibrillation. For determining the differences between the octogenarians group and those younger than 80 years, all variables with a $p<0.1$ on the univariate level were included into a multiple binominal regression analysis $(p$ to enter $=0.05, p$ to leave $=$ $0.1)$. Logistic regression models were used for dichotomous outcomes (e.g., $\mathrm{mRS} \geq 3$ and death). A value of $p<0.05$ was considered significant. Statistical analyses were performed using a statistical software package (Statistical Package for the Social Sciences, Version 17.0; SPSS, Chicago, IL).

\section{Results}

\section{Baseline Characteristics}

In Table 1, we show the clinical characteristics of the present cohort, comparing between octogenarians and younger patients. Hypertension, atrial fibrillation, and oral anticoagulation were more frequent in the octogenarian patients than in those patients younger than 80 years $(88.2$ vs. $65.5 \%, p=0.01 ; 73.5$ vs. $44.8 \%, p=0.003$; and 23.5 vs. $10.3 \%, p=0.04$, respectively). Smoking habit was more frequent in the younger patients (32.8 vs. $8.8 \%, p=$ $0.005)$. Diabetes mellitus, coronary disease, and dyslipemia showed no statistical differences between both groups. No differences were either detected regarding stroke subtype $(p=0.8)$, location of the occluded vessel $(p=0.7)$, previous intravenous thrombolysis (32.4 vs. $48.3 \%, p=0.1)$, and preprocedural NIHSS (18.1 vs. $16.8 \%, p=0.3$ )

\section{Procedure Data}

In Table 2, procedure and clinical outcome data are shown. There were no differences in the procedure times between both groups (63 (38-92) minutes in those younger than 80 years and 74.5 (40-114) minutes in the octogenarian patients, $p=0.2)$. Revascularization times also showed no difference between both groups (350 (296.3-452.8) minutes and 380.5 (298-526.3) minutes, respectively, $p=0.3)$. Time from symptom onset to groin puncture was 280 (235-365) minutes in patients younger than 80 years and 298 (247.5-421.3) minutes in octogenarians $(p=0.2)$. Postprocedure TICI score $\geq 2 \mathrm{~B}$ was reached in $93.9 \%$ of the patients younger than 80 , and $88.2 \%$ of the octogenarians, 
Table 1 Baseline characteristics of octogenarians and younger patients
EICA extracranial internal carotid artery, IICA intracranial internal carotid artery, $M C A$ middle cerebral artery, $T O$ tandem occlusion, VB vertebrobasilar, ATS atherothrombotic, $C E$ cardioembolic, $A D$ arterial dissection, $U N$ undetermined, NIHSS National Institute of Health Stroke Scale

Table 2 Procedure data and clinical outcome

\begin{tabular}{|c|c|c|c|}
\hline & $<80$ years $(n, \%)$ & $\geq 80$ years $(n, \%)$ & $p$ value \\
\hline Male sex & $66(56.9)$ & $12(35.3)$ & 0.03 \\
\hline High blood pressure & $76(65.5)$ & $30(88.2)$ & 0.01 \\
\hline Diabetes mellitus & $33(28.4)$ & $11(32.4)$ & 0.7 \\
\hline Coronary disease & $14(12.1)$ & $5(14.7)$ & 0.7 \\
\hline Atrial fibrillation & $52(44.8)$ & $25(73.5)$ & 0.003 \\
\hline Dyslipemia & $45(38.8)$ & $16(47.1)$ & 0.4 \\
\hline Smoking & $38(32.8)$ & $3(8.8)$ & 0.005 \\
\hline Anticoagulants & $12(10.3)$ & $8(23.5)$ & 0.04 \\
\hline \multirow[t]{5}{*}{ Occluded vessel } & EICA 8 (6.9) & EICA 4 (11.8) & 0.7 \\
\hline & IICA $21(18.1)$ & IICA 8 (23.5) & \\
\hline & MCA 55 (47.4) & MCA 13 (38.2) & \\
\hline & TO 15 (12.9) & TO $3(8.8)$ & \\
\hline & VB 17 (14.7) & VB 6 (17.7) & \\
\hline \multirow[t]{4}{*}{ Stroke subtype } & AT 39 (33.6) & AT $11(32.4)$ & 0.8 \\
\hline & CE 44 (37.9) & CE $14(41.2)$ & \\
\hline & AD 3 (2.6) & $\mathrm{AD} 0$ & \\
\hline & UN 30 (25.9) & UN 9 (26.5) & \\
\hline Previous IV thrombolysis & $56(48.3)$ & $11(32.4)$ & 0.1 \\
\hline Preprocedure NIHSS & 16.8 & 18.1 & 0.3 \\
\hline
\end{tabular}

NIHSS National Institute of Health Stroke Scale, $m R S$ modified Rankin Scale, TICI thrombolysis in cerebral infarction

\begin{tabular}{lllc}
\hline & $<80$ years $(n, \%)$ & $\geq 80$ years $(n, \%)$ & $p$ value \\
\hline NIHSS at discharge & 6.5 & 9.7 & 0.03 \\
Time of procedure & $63(38-92)$ & $74.5(40-114)$ & 0.2 \\
Time of revascularization & $350(296.3-452.8)$ & $380.5(298-526.3)$ & 0.3 \\
Time from symptoms onset to groin puncture & $280(235-365)$ & $298(247.5-421.3)$ & 0.2 \\
Postprocedure TICI $\geq 2 B$ & $109(93.9)$ & $30(88.2)$ & 0.1 \\
Symptomatic haemorrhage & $3(2.6)$ & $2(5.9)$ & 0.3 \\
mRS $\geq 3$ & $44 / 102(43.1)$ & $25 / 29(86.2)$ & $<0.0001$ \\
mRS 3-5 & $23 / 102(22.5)$ & $13 / 29(44.8)$ & 0.03 \\
Death & $20 / 150(17.2)$ & $12 / 150(35.3)$ & 0.02 \\
Causes of death & SP 14 (70) & SP8 $(66)$ & 0.08 \\
& VR 0 & VR 1 (8.3) & \\
& SH 2 (10) & SH 2 (16.7) & \\
\hline
\end{tabular}

octogenarians patients than in the younger group (35.3 vs. $17.2 \%, p=0.02)$. In the octogenarians patients group, deaths were secondary to stroke progression (defined as a worsening of NIHSS $\geq 4-15)$ in eight cases $(66.7 \%$ ), symptomatic haemorrhage in two (16.7\%), and systemic complication (pneumonia) and vessel rupture in one case each $(8.3 \%)$. In those patients younger than 80 , deaths were caused by stroke progression in 14 cases $(70 \%)$, systemic complications in $4(20 \%$, corresponding to 1 pulmonary thromboembolism, 1 acute renal failure, and 2 sepsis of urinary and pulmonary origin), and 2 symptomatic haemorrhages $(10 \%)$. There were no statistically significant differences between both groups $(p=0.08)$.
Postprocedural NIHSS was statistically higher in the octogenarians group than in younger patients (9.7 and 6.5, $p=0.03$ ). Death rate was more frequent in the 
Table 3 Multivariate analysis for mRS and death

\begin{tabular}{|c|c|c|c|c|c|}
\hline & $p$ & Odds ratio $(95 \% \mathrm{CI})$ & & $p$ & Odds ratio $(95 \% \mathrm{CI})$ \\
\hline mRS 3-5 AT 3 months & & & Death & & \\
\hline Male sex & 0.9 & $0.92(0.4-2.2)$ & Male sex & 0.4 & $1.45(0.6-3.5)$ \\
\hline High blood pressure & 0.01 & $5.05(1.4-18.4)$ & High blood pressure & 0.3 & $1.73(0.6-4.8)$ \\
\hline Atrial fibrillation & 0.6 & $0.8(0.3-19.3)$ & Atrial fibrillation & 0.3 & $1.54(0.6-3.8)$ \\
\hline Smoking & 0.06 & $0.31(0.1-1.1)$ & Smoking & 0.8 & $1.18(0.4-3.5)$ \\
\hline Oral anticoagulation & 0.7 & $0.82(0.3-2.7)$ & Oral anticoagulation & 0.3 & $1.73(0.6-4.9)$ \\
\hline
\end{tabular}

NIHSS National Institute of Health Stroke Scale, $m R S$ modified Rankin Scale, $S H$ symptomatic haemorrhage, $S P$ stroke progression, $S C$ systemic complication, TICI thrombolysis in cerebral infarction, $V R$ vessel rupture

Table 4 Subgroup analysis by occluded vessel and time of revascularization

\begin{tabular}{llll}
\hline & $<80$ years old & $\geq 80$ years old & $p$ \\
\hline $\begin{array}{lll}\text { Occluded vessel } \\
\text { ICA (extra or intracranial) }\end{array}$ & 29 & 12 & 0.23 \\
$\quad$ Other vessels & 87 & 22 & \\
Carotid occlusion & & & \\
mRS $\geq 3$ & $2 / 22(9.1)$ & $2 / 10(20.0)$ & 0.56 \\
Death & $8 / 29(27.6)$ & $8 / 12(66.7)$ & 0.03 \\
Other vessels & & & \\
mRS $\geq 3$ & $21 / 78(26.9)$ & $11 / 19(57.8)$ & 0.01 \\
$\quad$ Death & $12 / 87(13.8)$ & $4 / 22(18.2)$ & 0.6 \\
Time of revascularization & & & \\
$<6$ hours & 56 & 10 & 0.21 \\
$>6$ hours & 48 & 20 & \\
$<6$ hours & & & \\
mRS $\geq 3$ & $13 / 50(26.0)$ & $5 / 9(55.6)$ & 0.12 \\
Death & $9 / 56(16.1)$ & $2 / 11(18.2)$ & 1.00 \\
$>6$ hours & & & \\
mRS $\geq 3$ & $10 / 41(24.4)$ & $8 / 16(50.0)$ & 0.06 \\
Death & $9 / 48(18.6)$ & $6 / 19(31.6)$ & 0.25 \\
\hline
\end{tabular}

ICA internal carotid artery, $m R S$ modified Rankin Scale

A modified Rankin Scale at 3 months $\geq 3$ was significantly more frequent in the octogenarians group than in those younger than 80 years of age ( 86.2 vs. $43.1 \%, p<$ 0.0001). The effect of thrombectomy on survivors also was studied by performing a new analysis in which those patients with modified Rankin Scale $=6$ (death) were excluded. In those patients with a 3-month modified Rankin Scale $=3-5$, a statistical difference between octogenarians and younger patients also was found $(p=0.03)$.

All variables with a $p<0.1$ on the univariate level (high blood pressure, atrial fibrillation, oral anticoagulation, and smoking) were included into a multiple logistic regression analysis (Table 3). A previous history of hypertension independently associated to a modified Rankin Scale 3-5 at 3 months $(p=0.01)$.
As shown in Table 4, no differences in the distribution of ICA/other vessels were found between both groups ( $p=$ $0.23)$. However, ICA occlusion was associated with higher mortality in octogenarians than in younger patients $(p=$ $0.03)$, without differences in the 3-month mRS $(p=0.56)$. Regarding the time of revascularization, no differences in the distribution below or beyond 6 hours were found between both groups $(p=0.21)$. A trend to a worse mRS at 3 months was observed in those octogenarians treated beyond 6 hours, but it did not reach statistical significance $(p=0.06)$. There was no difference in mortality $(p=0.25)$.

\section{Discussion}

The purpose of our study was to test the effect of mechanical thrombectomy with stent-retrievers in octogenarians with acute ischemic stroke. Our results indicate that the use of these devices achieve good rates of recanalization in elderly patients $(88.2 \%)$ but still with a remarkably high mortality rate $(35.3 \%)$ and poor clinical prognosis (NIHSS at discharge, 3 months mRS) compared with younger patients.

Previous studies have already shown that poor outcomes after ischemic stroke are more frequent in octogenarians than in younger patients and that age $\geq 80$ years is a predictor of higher mortality and poorer functional outcome compared with younger patients after intravenous thrombolysis [2]. In the field of the endovascular therapy prior to stent-retrievers (Table 5), Kim et al. [3] obtained a recanalization rate of $68 \%$ by using intraarterial thrombolysis with urokinase or tPA, with a mortality of $43 \%$ and a tendency to higher mortality rates and lower functional outcomes in the octogenarians group compared with younger patients. Loh et al. [4] reported recanalization rates of $81 \%$ and a mortality of $48 \%$ in acute stroke octogenarians treated with the Merci thrombectomy device. Mono et al. [18] treated 53 patients with intra-arterial thrombolytics plus optional mechanical therapy with aspiration, clot retrieval, angioplasty, and/or stenting, with 
Table 5 Endovascular treatment of acute stroke in octogenarians. Most relevant previous studies and current series

\begin{tabular}{|c|c|c|c|c|c|}
\hline Type of endovascular therapy & $\begin{array}{l}\text { Number of } \\
\text { patients } \geq 80\end{array}$ & $\mathrm{TICI} \geq 2 \mathrm{~B}$ & $\begin{array}{l}\text { Symptomatic } \\
\text { Hemorrhage }\end{array}$ & $\mathrm{mRS} \geq 3$ & Mortality \\
\hline UK, rTPA (3) & 33 & $68 \%$ & $7 \%$ & $73 \%$ & $43 \%$ \\
\hline UK, rTPA, AS- AP- ST (18) & 53 & $65 \%$ & $6 \%$ & $72 \%$ & $40 \%$ \\
\hline Classic retrievers: Merci (4) & 31 & $81 \%$ & $11 \%$ & $79 \%$ & $48 \%$ \\
\hline Classic retrievers: Merci, Penumbra (19) & 34 & $71 \%$ & $4 \%$ & $97 \%$ & $58 \%$ \\
\hline Stent-retrievers: Solitaire FR (7) & 50 & $74 \%$ & $14 \%$ & $89 \%$ & $44 \%$ \\
\hline Stent-retrievers: Solitaire FR, Trevo, Capture (our center) & 34 & $88 \%$ & $5 \%$ & $73 \%$ & $35 \%$ \\
\hline
\end{tabular}

TICI thrombolysis in cerebral infarction; $m R S$ modified Rankin Scale; $U K$ uroquinase; $r T P A$ recombinant tissue plasminogen activator $M R$ mechanical recanalization; $A S$ clot aspiration; $A P$ angioplasty' $S T$ stenting

similar results, and Chandra et al. [19] reviewed 34 patients treated with the Merci retriever or the Penumbra aspiration device, finding a mortality of $58 \%$ and $\mathrm{mRS} \geq 3$ at 3 months of $97 \%$. Previous studies with stent retrievers in patients older than 80 years include Gratz et al. [7], who studied 50 octogenarian patients with anterior circulation strokes treated exclusively with Solitaire FR, with a recanalization rate of $74 \%, \mathrm{mRS} \geq 3$ at 3 months of $89 \%$, and mortality of $44 \%$. Other articles analyzed the general impact of age in the clinical response to mechanical thrombectomy [20-22], but these papers did not focus on the subgroup of octogenarians and included other mechanical therapies different to stent-retrievers.

Our series differ from Gratz's in that both patients with anterior and posterior circulation strokes have been included and up to four different stent-retrievers (not only Solitaire FR) could be chosen for treatment by the neurointerventionist in charge. However, the similar results obtained seem to confirm that, despite the high rates of recanalization, thrombectomy with stent retrievers in octogenarians are still associated with high mortality and morbidity rates. This bad clinical evolution is probably due to the vascular fragility of these patients, which is favoured by age and the higher incidence of related risk factors, but some modifiable factors also might be influencing.

In this sense, our results suggest that three main factors may influence the worst prognosis observed in the group of octogenarians: (1) higher incidence of risk factors, such as high blood pressure $(p=0.01)$, atrial fibrillation $(p=$ $0.003)$, or anticoagulants intake ( $p=0.04),(2)$ the location of the occluded vessel (ICA occlusion associate to higher mortality in octogenarians than in younger patients $(p=$ 0.034 ), and (3) the revascularization time, which shows an association trend with worst 3-month mRS in those octogenarians treated beyond 6 hours (Table 3 ). This seems to indicate that these three conditions (associated cardiovascular risk factors, ICA involvement, and revascularization time $>6$ hours) are especially harmful for the elderly patients and could be taken into account to adapt selection criteria and procedural and postprocedural management approaches and avoid futile recanalization in this group of age.

The main drawbacks of our study are related with the prolonged revascularization times, which are due to the big area/population covered by our centre and an inefficient patient transfer system, which is currently being improved with measures, such as a bigger mobile unit's fleet or a 24-hour stroke ambulance located in each of the six big main hospitals of the region. These prolonged times are observed in both arms of the study (octogenarians and younger patients, $p=0.4$ ), which makes the differences found in mortality and clinical outcome still of value.

Also of interest, our procedure times seem to be slightly beyond what is considered optimal. At our centre, stroke thrombectomies are performed under general anaesthesia, a situation that allows us to work in a more controlled situation but may carry a longer preparation of the patient. Our current results seem to indicate that sedation might be a better option to save critical time in the elderly patients. Prospective studies are needed to test this and the previous aspects suggested by our results.

\section{Conclusions}

In our series, treatment with stent-retrievers in octogenarians with acute ischemic stroke achieved good rates of recanalization but worse clinical outcome than younger patients. A higher incidence of risk factors, ICA involvement, and revascularization times beyond 6 hours were associated to a worse prognosis. These data might be of value in the design of prospective studies evaluating the clinical efficacy of the endovascular treatments in octogenarians.

Conflict of interest All authors declare that they have no conflict of interest.

Sources of funding None. 


\section{References}

1. Hassan AE, Chaudry SA, Grigoryan M et al (2012) National trends in utilization and outcomes of endovascular treatment of acute ischemic stroke patients in the mechanical thrombectomy era. Stroke 43:3012-3017

2. Mishra NK, Ahmed N, Andersen G et al (2010) Thrombolysis in very octogenarians people: controlled comparison of SITS International Stroke Thrombolysis Registry and Virtual International Stroke Trials Archive. BMJ 341:c6046. doi:10.1136/bmj. c6046

3. Kim D, Ford GA, Kidwell CS et al (2007) Intra-arterial thrombolysis for acute stroke in patients 80 and older: a comparison of results in patients younger than 80 years. Am J Neuroradiol 28:159-163

4. Loh Y, Kim D, Shi ZS et al (2010) Higher rates of mortality but not morbidity follow intracranial mechanical thrombectomy in the octogenarians. Am J Neuroradiol 31:1181-1185

5. Novakovic RL, Toth G, Narayanan S et al (2012) Retrievable stents, "stentrievers," for endovascular acute ischemic stroke therapy. Neurology 79(13 Suppl 1):S148-S157

6. Saver JL, Jahan R, Levy EI et al (2012) Solitaire flow restoration device versus the Merci Retriever in patients with acute ischemic stroke (SWIFT): a randomised, parallel-group, non-inferiority trial. Lancet 380:1241-1249

7. Gratz PP, Jung S, Schroth G et al (2013) Outcome of standard and high risk patients with acute anterior circulation stroke after stent retriever thrombectomy. Stroke Nov 21 (Epub ahead of print)

8. (1995) Tissue plasminogen activator for acute ischemic stroke: the National Institute of Neurological Disorders and Stroke rt-PA Stroke Study Group. N Engl J Med 333:1581-1587

9. Wahlgren N, Ahmed N, Davalos A et al (2008) Thrombolysis with alteplase 3-4.5 h after acute ischaemic stroke (SITS-ISTR): an observational study. Lancet 372:1303-1309

10. Hacke W, Kaste M, Bluhmki E et al (2008) Thrombolysis with alteplase 3 to 4.5 hours after acute ischemic stroke. New Engl J Med 359:1317-1329

11. Smith WS, Sung G, Starkman S et al (2005) Safety and efficacy of mechanical embolectomy in acute ischemic stroke: results of the MERCI trial. Stroke 36:1432-1438
12. Smith WS, Sung G, Saver J et al (2008) Mechanical thrombectomy for acute ischemic stroke: final results of the Multi MERCI trial. Stroke 39:1205-1212

13. Castaño C, Dorado L, Guerrero C et al (2010) Mechanical thrombectomy with the Solitaire $\mathrm{AB}$ device in large artery occlusions of the anterior circulation: a pilot study. Stroke 41:1836-1840

14. Wehrschuetz M, Wehrschuetz E, Augustin M et al (2011) Early single-center experience with the Solitaire thrombectomy device for the treatment of acute ischemic stroke. Interv Neuroradiol $17: 235-240$

15. Kan PT, Orion D, Yashar P et al (2011) Intra-arterial thrombolysis and thrombectomy for acute ischemic stroke: technique and results. J Neurosurg Sci 55:151-160

16. Higashida RT, Furlan AJ, Roberts H et al (2003) Trial design and reporting standards for intraarterial cerebral thrombolysis for acute ischemic stroke. Stroke 34:109-137

17. Adams HP, del Zoppo G, Alberts HJ et al (2007) Guidelines for the early management of adults with ischemic stroke. Circulation 115:478-534

18. Mono ML, Romagna L, Jung S et al (2012) Intra-arterial thrombolysis for acute ischemic stroke in octogenarians. Cerebrovasc dis 33:116-122

19. Chandra R, Leslie-Mazwi TM, Oh D et al (2012) Elderly patients are at higher risk for poor outcomes after intra-arterial therapy. Stroke 43:2356-2361

20. Raoult H, Eugene F, Ferre JC et al (2013) Prognostic factors for outcomes after mechanical thrombectomy with Solitaire stent. J Neuroradiol 40:252-259

21. Kurre W, Aguilar-Perez M, Niehaus L et al (2013) Predictors of outcome after mechanical thrombectomy for anterior circulation large vessel occlusion in patients aged over 80 years. Cerebrovasc Dis 36:430-436

22. Singer OC, Haring HP, Trenkler J et al (2013) Age dependency of successful recanalization in anterior circulation stroke: The ENDOSTROKE study. Cerebrovasc Dis 36:437-455 